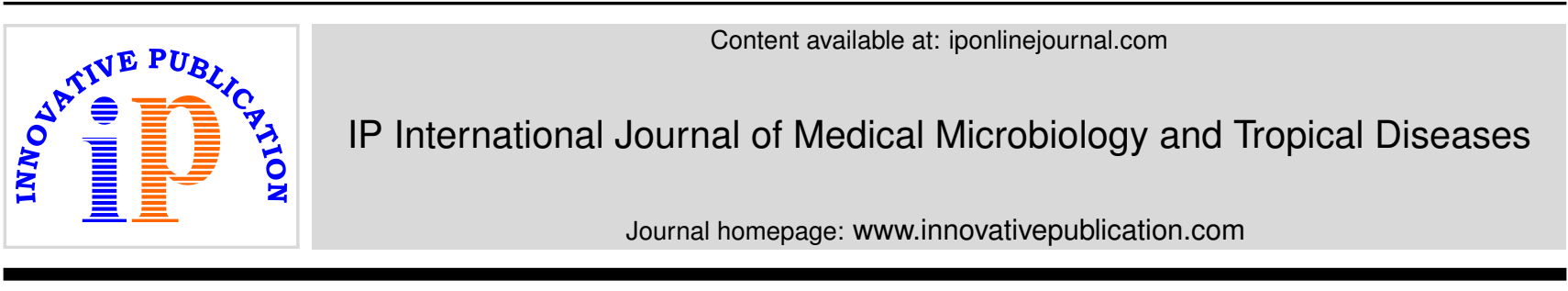

Original Research Article

\title{
Study of prevalence Rifampicin and/ or Isoniazid drug resistance among newly diagnosed cases of sputum smear positive pulmonary tuberculosis at tertiary care centre
}

\author{
Sushant Dilip Muley ${ }^{1} *$, Sushant Meshram ${ }^{1}, \operatorname{Ravi}_{\text {Yadav }^{1}}$, Tauseef Naeem ${ }^{1}$, \\ Avinash Gandhare ${ }^{1}$ \\ ${ }^{1}$ Dept. of Pulmonary Medicine, Govt. Medical College, Nagpur, Maharashtra, India
}

\section{A R T I C L E I N F O}

\section{Article history:}

Received 15-01-2020

Accepted 12-03-2020

Available online 26-04-2020

\section{Keywords:}

multidrug resistant

extensively drug resistant isoniazid monoresistance India

\begin{abstract}
A B S T R A C T
Introduction: Worldwide tuberculosis is most common infectious cause of death. India bears one-fourth of the global tuberculosis burden. Despite all national \& international efforts to control \& eliminate tuberculosis, more than 10 million active tuberculosis cases occur each year. The problem of tuberculosis has been further compounded by the emergence of multi drug resistant (MDR) and extensively drug resistant(XDR) tuberculosis. Previously treated tuberculosis is most important risk factor for drug resistant TB but treatment naïve patients are also at risk due to transmission of drug resistant strain or spontaneous mutation.

Materials and Methods: Study was conducted in Department Of Respiratory Medicine, GMC Nagpur between May 2017 to October 2018.It was prospective observational study. All sputum smear positive cases were subjected to mycobacterial culture and LPA testing in IRL.

Results: Total 250 patients whose sputum smear was positive for acid fast bacilli were included in study. Maximum patients were in age group of 18-30 years of age with mean age 36.43+/-14.05. Out of 250 patient 188 were males and 62 were females. Isoniazid monoresistance was found in 6 patients and isoniazid with rifampicin resistance was found in 9 patients. We did not find any case of rifampicin monoresistance in our study.

Conclusion: Prevalence of isoniazid monoresistance was $2.4 \%$ in our study while prevalence of isoniazid and rifampicin resistance (MDR TB) was 3.6\%. Thus prevalence of drug resistant TB is low amongst newly diagnosed sputum smear positive cases. This shows success of tuberculosis control programme.
\end{abstract}

(C) 2020 Published by Innovative Publication. This is an open access article under the CC BY-NC-ND license (https://creativecommons.org/licenses/by/4.0/)

\section{Introduction}

Drug-resistant pulmonary tuberculosis (DR-TB) is a major public health problem that considerably affects the present TB control programme in India. Tuberculosis (TB) control efforts are threatened by the emergence of Mycobacterium tuberculosis strains that are resistant to first-line and second line drugs. In 2017, TB was responsible for an estimated 1.3 million deaths among HIV-negative people, and an additional 300000 deaths from TB among HIVpositive people. There were an estimated 10.0 million

\footnotetext{
* Corresponding author.

E-mail address: muley.sushant@yahoo.in (S. D. Muley).
}

new cases of TB, equivalent to 133 cases per 100000 population. Globally in 2017, there were an estimated 558 000 new cases of rifampicin resistant TB (RR-TB), of which almost half were in three countries: India (24\%), China (13\%) and the Russian Federation (10\%). Among RR-TB cases, an estimated $82 \%$ had multidrug-resistant TB (MDR-TB). Globally, $3.5 \%$ of new TB cases and $18 \%$ of previously treated cases had MDR/RR-TB, with the highest proportions ( $>50 \%$ in previously treated cases) in countries of the former Soviet Union. Previous treatment for TB is the most important risk factor for development of MDR-TB, but naïve patients are also at risk due to either spontaneous mutations or transmission of drug-resistant strains. ${ }^{1}$ The 
risk of transmission of resistant strains from close contacts is increasing because of the growing burden of MDRTB patients. Therefore, there is high likelihood that what initially seems to be drug-sensitive TB in a treatment-naïve patient might in fact be MDR TB. Therefore, there is a need to determine the prevalence of MDR-TB among new cases of sputum-positive pulmonary TB

\section{Materials and Methods}

This study was conducted in department of respiratory medicine , Government medical college , Nagpur which is also a nodal DR TB centre attached to IRL.

\subsection{Study design}

Hospital based prospective observational study

\subsection{Study sample}

All the patients presenting to or referred to Respiratory Medicine OPD at tertiary care center as a newly diagnosed case of sputum AFB smear positive pulmonary tuberculosis.

\subsection{Study period}

May 2017 to October 2018

\subsection{Sampling method}

The patients selected were newly diagnosed sputum AFB smear positive pulmonary tuberculosis from outpatient and inpatient of department of Respiratory Medicine of same institute. The study was carried out after approval from the institutional ethical committee and with fully informed and written consent from the subjects. All sputum smear positive cases were subjected to mycobacterial culture and LPA testing in IRL.

\subsection{Statistical Analysis}

Data was entered in Microsoft excel 2010 and analysed. The proportions of study variables were calculated and expressed in terms of percentages.

\section{Results}

Maximum patients were in age group of 18-30 years followed by 31-40 years

Almost all patient had BMI less than 25 which suggest undernutrition.

\subsection{Drug resistance pattern}

6 patient had only isoniazid resistance while 9 patients were positive for both isoniazid and rifampicin resistance.

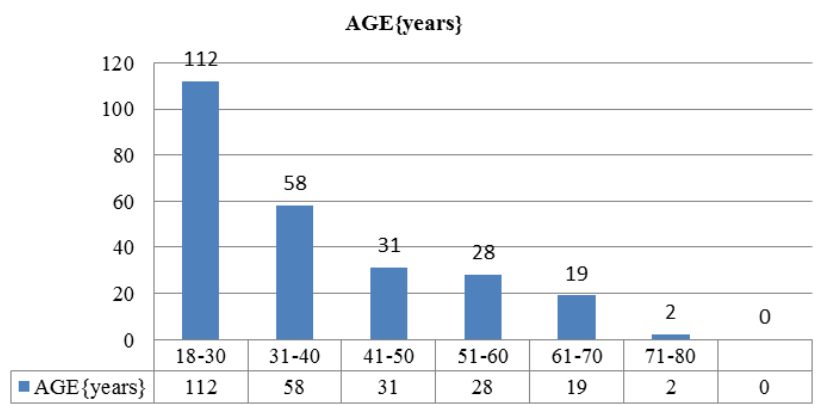

Fig. 1: Distribution of patients according to age

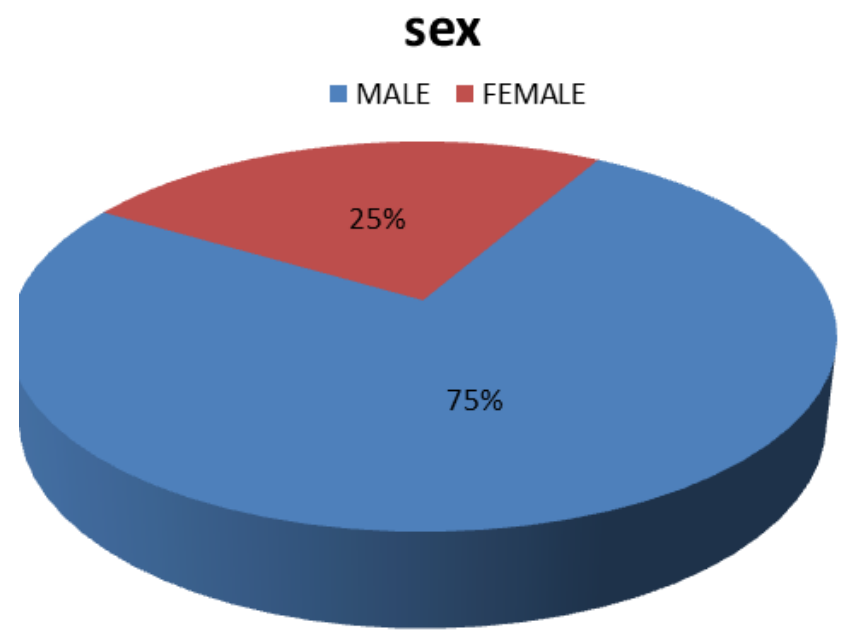

Fig. 2: Distribution of patients according to Sex

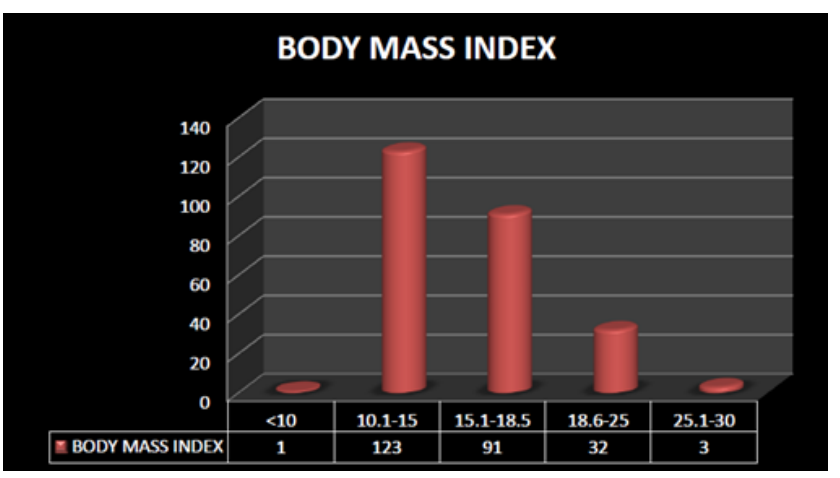

Fig. 3: Distribution of patients according to body mass index

\section{Discussion}

The present study was prospective observational study conducted in department of respiratory medicine, Government medical college, Nagpur to find out prevalence of drug resistance in newly diagnosed sputum smear positive cases. The most cost effective measure to treat the drug susceptible tuberculosis is provision and implementation of good quality DOTS thereby preventing the spread of 


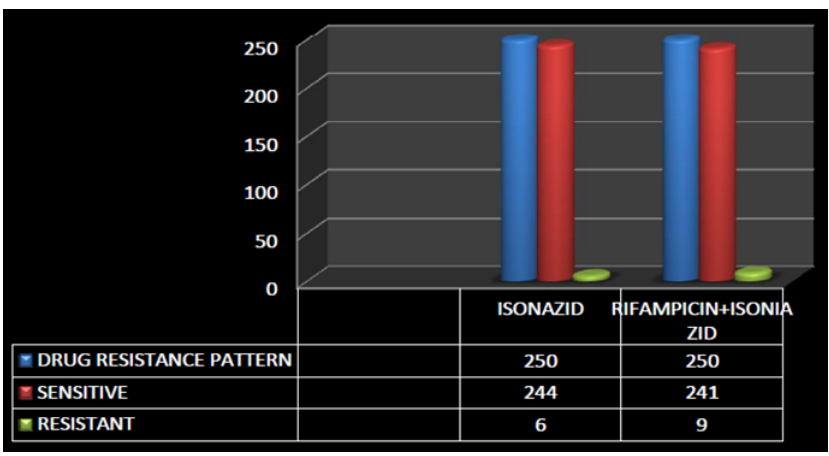

Fig. 4: Ditribution of patients according to drug resistance pattern

emergence of drug resistant tuberculosis. Maximum patients were in age group of 18-30 years followed by 31-40 years (Figure 1). The mean age of patient was 36.43(+/- 14.05) years. This demographic profile was similar to other studies by Rawat $\mathrm{J}$ et al, ${ }^{2}$ Ajaz et al ${ }^{3}$ and V.Agwan et al. ${ }^{4}$ There were greater number of male patients than female patients in this study with $75.2 \%$ males and $24.8 \%$ females (Figure 2). These results were similar with studies by SK Sharma et al ${ }^{5}$ Jethani $\mathrm{S}$ etal. ${ }^{6}$

In this study, about $86 \%$ patients were malnourished (Figure 3),12.8\% patients were in the normal range, 1.2\% were pre obese, with median BMI $(15.38+/-3.51)$. Kasim $\mathrm{M}$ et $\mathrm{al}^{7}$ studied the nutritional status and body mass index of pulmonary tuberculosis patients and found that mean BMI of pulmonary tuberculosis cases was $(16.9 \pm$ $1.1 \mathrm{~kg} / \mathrm{m} 2$ ). Natasha S. Hochberg et $\mathrm{al}^{8}$ found that $60.2 \%$ of tuberculosis patients were malnourished (body mass index $[\mathrm{BMI}]<18.5 \mathrm{~kg} / \mathrm{m} 2)$. We found 6 patients $(2.4 \%)$ with isoniazid monoresistance and 9 patients $(3.6 \%)$ with both isoniazid and rifampicin resistance (Figure 4). This results are comparable with Indian national figures of 2.84 $\%$ of primary MDR TB per PMDT guiselines $2017 .{ }^{9}$ While prevalence of isoniazid monoresistance was $11.6 \%$ in newly detected sputum smear positive patients as per 2017 PMDT guidelines, it was very low in our study $(2.4 \%)$. This may be the result of timely diagnosis and treatment of tuberculosis and successful implementation of programme.

\section{Source of funding}

None.

\section{Conflict of interest}

None.

\section{References}

1. Sharma SK, Kumar S, Saha PK. Prevalence of multidrug-resistant tuberculosis among category II pulmonary tuberculosis patients. Indian J Med Res. 2011;133(3):312-315.

2. Rawat J, Sindhwani G, Juyal R. clinico-radiological profile of new smear positive pulmonary tuberculosis cases among young adult and elderly people in a tertiary care hospital at deheradun (uttarakhand). Indian J Tub. 2008;55:84-90.

3. Koul AN, Wagay HA. Demography and clinical outcome of pulmonary tuberculosis in kashmir; 2 year prospective study. Egyptian J Chest Dis Tuberculosis. 2015;

4. Agwan V, Kansal R, Madan M, Asthana AK. Primary multi drug resistance in new pulmonary tuberculosis patients in western uttar pradesh, india. Int J Curr Microbiol App Sci. 2015;4(8):656-663.

5. Sharma SK, Kaushik G, Jha B. Prevalence of multidrugresistant tuberculosis among newly diagnosed cases of sputum-positive pulmonary tuberculosis. Indian J Med Res. 2011;133(3):308-311.

6. Jethani S, Kakkar R, Semwal J, Rawat J. Demographic Profile of Tuberculosis patient: A hospital based study at Dehradun. Natl $J$ Community Med. 2014;5(1):6-9.

7. Sultan KM, Alobaidy MW, AL-Jubouri AM, Naser AA, AL-Sabah HA Assessment of Body Mass Index and Nutritional Status in Pulmonary Tuberculosis Patients. J Fac Med Baghdad. 2012;54(3).

8. Hochberg NS, Sarkar S, Horsburgh CR, Knudsen S, Pleskunas J, et al. Comorbidities in pulmonary tuberculosis cases in Puducherry and Tamil Nadu, India: Opportunities for intervention. PLOS ONE. 2017;12(8):e0183195-e0183195.

9. PMDT Guidelines; 2017.

\section{Author biography}

Sushant Dilip Muley Assistant Professor

Sushant Meshram Professor and Head

Ravi Yadav Assistant Professor

Tauseef Naeem Junior Lecturer

Avinash Gandhare Intern Resident

Cite this article: Muley SD, Meshram S, Yadav R, Naeem T, Gandhare A. Study of prevalence Rifampicin and/ or Isoniazid drug resistance among newly diagnosed cases of sputum smear positive pulmonary tuberculosis at tertiary care centre. IP Int J Med Microbiol Trop Dis 2020;6(1):39-41. 